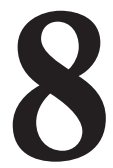

\title{
EDUCACIÓN SUPERIOR VIRTUAL EN COLOMBIA: FACTORES ASOCIADOS AL ABANDONO
}

\section{(VIRTUAL HIGHER EDUCATION IN COLOMBIA: FACTORS ASSOCIATED WITH DROPPING OUT)}

Nuria Segovia-García

Corporación Universitaria de Asturias

Elías Said-Hung

Francisco José García Aguilera

Universidad Internacional de La Rioja

DOI: 10.5944/educXX1.30455

\section{Cómo referenciar este artículo/How to reference this article:}

Segovia-García, N., Said-Hung, E. y García Aguilera, F. (2022). Educación Superior virtual en Colombia: factores asociados al abandono. Educación XX1, 25(1), 197-218. https://doi.org/10.5944/ educXX1.30455

Segovia-García, N., Said-Hung, E., \& García Aguilera, F. (2022). Virtual Higher Education in Colombia: factors associated with dropping out. Educación XX1, 25(1), 197-218. https://doi. org/10.5944/educXX1.30455

\section{RESUMEN}

En los últimos años Colombia ha experimentado una fuerte demanda de estudiantes provenientes de una amplia diversidad de puntos geográficos y entornos socioeconómicos que quieren acceder al sistema de educación superior. Para garantizar la acogida de todos los estudiantes se han desarrollado estrategias que incrementan la oferta educativa y facilitan el acceso a través de modalidades novedosas como la virtual o a distancia virtual. Pero a pesar de las mejoras en la cobertura, Colombia sigue teniendo un reto importante en cuanto a la permanencia al registrar altas tasas de abandono. Este trabajo tiene como objetivo analizar los factores que están afectando al abandono de la educación superior en Colombia en modalidad virtual; para ello se ha diseñado una encuesta que recoge, a través de 62 ítems, información 
demográfica, socioeconómica, académica, de infraestructura tecnológica y de satisfacción. La muestra ha estado formada por 384 estudiantes con intención de abandono en alguno de los 403 programas de pregrado en modalidad virtual, con registro calificado activo a junio de 2019, según el SNIES. A partir de un estudio cuantitativo, en el que se empleó regresión logística multinomial como modelo estadístico, se ha podido establecer cómo, entre otras variables, la satisfacción que experimentan los estudiantes con relación a los servicios recibidos por parte de la institución y el contenido ofrecido para desarrollar su programa, son factores que están asociadas a la permanencia académica de estos. Por lo que el papel asumido por las Instituciones de Educación Superior, en garantizar la permanencia de los estudiantes, bajo modelos educativos virtuales, requiere de una respuesta educativa equitativa y de calidad, atendiendo especialmente a los estudiantes más vulnerables.

\section{PALABRAS CLAVE}

Aprendizaje en línea, educación superior, Colombia, abandono educativo, satisfacción de alumnos, análisis de regresión

\section{ABSTRACT}

Colombia has experienced a strong demand from students from a wide variety of geographical locations and socioeconomic backgrounds who want access to higher education. To ensure the reception of all students, strategies have been developed to increase the supply of education and facilitate access through innovative modalities such as virtual or distance learning. However, despite these improvements in the coverage of the educational system, high university dropout rate is a challenge for Colombia. This research focuses on analyzing the dropout factors in distance learning universities. For this purpose, a survey has been designed that collects, through 62 items, demographic, socio-economic, academic, technological infrastructure and satisfaction information. The sample consisted of 384 students with the intention of dropping out of any of 403 undergraduate programs in virtual modality, with active qualified registration as of June 2019, according to the SNIES. A quantitative research based on the logistic regression method was conducted and the results indicate how among other variables, student satisfaction with their university is associated with quality differences of offered services and academic content that are factors associated with student retention. The results allow to conclude that universities have a crucial role in order to guarantee continuation in education for all students, obliged to provide quality and equitable educational response with special attention to the most vulnerable population. 


\section{KEY WORDS}

Online learning, higher education, Colombia, dropping out, student satisfaction, regression analysis

\section{INTRODUCCIÓN}

Desde el inicio del siglo xxI y vinculado a un periodo de crecimiento económico y cambio político, los países de América Latina y el Caribe están experimentando una expansión de la educación superior que ha tenido como resultado la apertura de la universidad a alumnado de entornos socioeconómicos medios y bajos (Benavides et al., 2018). Esta apertura está introduciendo cambios notables en los diferentes sistemas educativos por la necesidad de facilitar el acceso a todos los estudiantes que lo deseen, pero, lo que es más importante, por la responsabilidad de garantizar la permanencia y graduación de estos, exigiendo una respuesta educativa personalizada y acorde con sus necesidades (Fernández y Pérez, 2016).

En el caso de Colombia, este crecimiento ha sido exponencial posicionándose como uno de los 25 países del mundo que mayor impulso ha tenido en el nivel terciario, con un incremento del $22.6 \%$ entre el periodo de 2012 a 2016 (Organización para la Cooperación y el Desarrollo Económico [OECD], 2019). Pero pese a estos avances el acceso a la educación superior sigue siendo limitado, con grandes diferencias entre el ámbito urbano y rural y además presentando altas tasas de deserción (Ministerio de Educación Nacional [MINEDUCACION], 2021), tal como muestran los datos registrados por el Sistema para la Prevención y Análisis de la Deserción en las Instituciones de Educación Superior (SPADIES), con corte de información en abril de 2020, situando la deserción en Colombia en un $35.6 \%$.

Uno de los problemas con los que cuenta Colombia a la hora de garantizar una adecuada cobertura y calidad de la educación superior es, por un lado, la dispersión geográfica de los estudiantes sobre todo los de las zonas rurales donde se ha demostrado que existen mayores barreras de acceso (Arias-Velandia et al., 2018) y por otro la tendencia a concentrar las universidades en las zonas urbanas con mayores recursos. Este dato se corrobora al advertir cómo de las 378 universidades con programas con registro calificado activo en 2020 (Sistema Nacional de Información de la Educación Superior [SNIES], s.f.), más de un tercio se encuentran en Bogotá D.C. y el resto se concentran en los departamentos de Antioquia, Valle del Cauca, Santander o Atlántico, teniendo una presencia muy baja o nula en departamentos como Vaupés, Guaviare, Guainía y Vichada. 
Con objeto de paliar las deficiencias anteriores, el Ministerio de Educación Nacional de Colombia (MEN) ha desplegado numerosas estrategias orientadas a aumentar la tasa de cobertura incrementando para ello el número de municipios de Colombia con oferta universitaria, creando nuevos Centros Regionales de Educación Superior (CERES) y fomentando el apoyo y acompañamiento de las Instituciones de Educación Superior (IES) para incorporar las Tecnologías de la Información y Comunicación (TIC) en sus procesos educativos (MINEDUCACIÓN, 2010). El resultado de estas acciones ha contribuido a que en los últimos años haya crecido de manera significativa el número de IES que ofrecen programas de educación terciaria, contabilizando en un cuarto de las que actualmente existen, siendo la mayoría de titularidad privada (258 privadas frente a las 118 oficiales) y que además se caracterizan por haber diversificado su modelo educativo promocionando el desarrollo de modalidades de formación alternativas como a distancia virtual o virtual (Fernández y Pérez, 2016; SNIES, s.f.).

A partir de lo expuesto hasta aquí, es posible destacar otra de las problemáticas señaladas por autores como Bonilla et al. (2015) y Gamboa y Rodríguez-Lesmes (2014) en cuanto a la educación superior, y es que el hecho de tener un desequilibrio tan marcado en la oferta de cupos en las IES públicas perjudica de manera directa la posibilidad de acceder a estas instituciones a estudiantes con mayores desventajas acumulativas. No hay que olvidar que el acceso a la educación superior viene supeditado por los criterios establecidos a partir del puntaje obtenido en el examen nacional de bachillerato (SABER 11), una prueba que beneficia a los estudiantes provenientes de escuelas secundarias privadas con mejores recursos y una mayor preparación (Bonilla et al., 2015; Gamboa y Rodríguez-Lesmes, 2014). Se delimita así otro de los factores clave en el acceso y permanencia de los estudiantes en la educación superior en Colombia, y es el relacionado con las dificultades económicas que experimentan los estudiantes de estratos socioeconómicos más bajos (1 y 2 fundamentalmente) (Melguizo et al., 2016; Munizaga et al., 2018). La falta de oferta pública adecuada, junto con el alto coste de algunas universidades privadas y la falta de un sistema de ayuda financiera bien desarrollado hace que en muchas ocasiones estos alumnos de estratos bajos deban acceder a universidades de titularidad privada de baja calidad donde los programas ofertados carecen de criterios adecuados en cuanto a calidad, pertinencia y empleabilidad, generando una deserción temprana al percibir una falta de retorno en la inversión que están acometiendo (Melguizo et al., 2016).

Lo anterior dibuja un panorama de la educación superior en Colombia complejo, y más a partir de la primera mitad del año 2020 con la llegada de la pandemia del SARS-CoV-2, que ha modificado de manera importante la dinámica de las IES obligándolas a adoptar modalidades de formación como 
la virtual para las que muchas no están preparadas y que en cuanto a los efectos causados en la sociedad y economía podrían agravar los problemas ya analizados para garantizar la retención y permanencia de los estudiantes. Estos precedentes han generado que en este trabajo se quiera profundizar sobre el fenómeno del abandono ofreciendo una respuesta sobre cuáles son los factores que están impactando en esta decisión, pero abordado desde modalidades como la virtual o a distancia virtual para las que aún no existe un amplio número de investigaciones que den cuenta de este fenómeno en Colombia.

\section{El abandono en la educación superior. Modelos de estudio}

Los datos proporcionados por el SPADIES sobre la tasa de abandono en la educación superior exigen realizar una reflexión crítica sobre este problema. Como punto de partida para el análisis del abandono en la educación superior es necesario recurrir a los modelos que tradicionalmente han dado respuesta desde un punto de vista integral. A partir de las aportaciones de autores como Cabrera et al. (2006) es posible obtener una agrupación de los diferentes enfoques adoptados a lo largo de la literatura para explicar el abandono, recogiendo cuatro modelos: adaptación, estructural, economicista y psicopedagógico.

El modelo de adaptación, referido a la incapacidad de los estudiantes de adaptarse a su medio académico toma como base el concepto de anomia, extraído de la sociología y generado a partir del pensamiento de autores como Durkheim y Merton (Pérez, 2017) y que transferido al ámbito educativo permeabiliza los problemas encontrados por los estudiantes para integrarse en su institución educativa o contexto social/académico. Este modelo ha sido la base para que Tinto (1987) desarrollara su teoría de la persistencia donde se establecía la relación directa del abandono con el grado de integración académica (rendimiento académico) y social (interacciones entre estudiantes, docentes y comunidad educativa) en su institución educativa. Además del anterior, que es el más empleado, otros modelos como el estructural y el economicista amplían el ámbito de estudio de los factores asociados al abandono introduciendo variables más estructurales que toman en cuenta las situaciones personales de los estudiantes en cuanto a estrato socioeconómico, salario, ocupación, políticas de apoyo financiero, entre otros (Cabrera et al., 2006; Fernández, 2016) o en el caso del economicista centrándose en los beneficios potenciales entre la inversión que ha de realizarse para acometer los estudios y el retorno que se va a obtener. Por su parte, el modelo psicopedagógico complementa los anteriores e integra variables más ligadas a la interacción psicopedagógica de los estudiantes (grado de satisfacción académica, estrategias de aprendizaje, habilidades de estudio, entre otras). 
Todos estos modelos contribuyen a generar un cuerpo de estudio sobre el fenómeno de abandono muy amplio, incorporando variables sociodemográficas, socioeconómicas, socioeducativas, académicas, institucionales, entre otras. Sin embargo, la mayoría de estos modelos se han gestado en entornos de aprendizaje presenciales donde la interacción con la institución, los materiales, el programa o los compañeros son radicalmente diferentes a cómo se genera en comunidades virtuales. En este sentido, cuando se trata de analizar el fenómeno del abandono en entornos virtuales es necesario contemplar otros modelos centrados en la calidad de los sistemas virtuales. Uno de los más representativos es el de DeLone y McLean (1992) para medir la calidad de los sistemas de información a través de seis categorías interdependientes (calidad de la información, calidad del sistema, satisfacción del usuario, uso del sistema, impacto individual e impacto organizativo) y que posteriormente se amplió a siete categorías de análisis al incorporar la calidad del servicio como elemento clave (DeLone y McLean, 2003). El modelo de DeLone y McLean (2003) ha sido la base para el desarrollo de numerosas investigaciones como las más recientes desarrolladas por Aldholay et al. (2018), Mustafa et al. (2020) o Salam y Farooq (2020), entre otros.

Lo anterior indica la necesidad de ampliar el marco de estudio sobre el abandono educativo complementando los modelos que tradicionalmente han dado respuesta a esta problemática e integrando nuevas variables que puedan dar una respuesta más certera a las principales razones que están generando estas cifras. Todo ello en un contexto mundial donde la virtualidad se está posicionando como una modalidad necesaria para dar alcance a las metas de la educación superior en cuanto a la mejora de la cobertura, y provisión de una educación de calidad que garantice la permanencia y graduación de los estudiantes y por tanto se requiere una mayor comprensión de las variables que amenazan la pertinencia y calidad del modelo.

Tomando como base lo expuesto hasta aquí nuestra investigación se va a centrar en detectar cuáles son los factores que están impactando de manera importante en el abandono de la educación superior en modalidad virtual y que contrarrestan significativamente el alcance de las metas de cobertura y calidad planificadas por el MEN. Las hipótesis sobre las que va a girar este trabajo son las siguientes:

- H1: Existe una asociación estadística fuerte entre el abandono de los estudios y las variables socioeconómicas de los estudiantes.

- H2: Existe una asociación estadística fuerte entre el abandono de los estudios y las variables sociodemográficas de los estudiantes. 
- H3: La percepción de una baja calidad del servicio prestado por la institución en cuanto a procesos administrativos, acompañamiento y mejora educativa influye de manera determinante en la decisión de abandonar el programa.

- H4: La calidad percibida por parte de los estudiantes en cuanto a infraestructura tecnológica prestada por la institución para desarrollar los programas virtuales tiene una influencia directa y significativa sobre la decisión de permanecer o abandonar los estudios en modalidad virtual.

- H5: La calidad del plan de estudios en cuanto a la pertinencia, secuencia y actualización de los contenidos ejerce una influencia significativa en la decisión de abandono de los estudios.

- H6: La calidad de las interacciones generadas entre docentes y compañeros es un elemento que influye en la decisión de abandonar los estudios.

\section{METODOLOGÍA}

\section{Participantes}

La población ha estado conformada por los 208784 estudiantes que según los datos registrados por el SNIES (2019) estaban matriculados en alguno de los 403 programas de pregrado virtual ofertados en Colombia y que cuentan con registro calificado a la fecha de desarrollar el estudio. Se ha distribuido una encuesta a través de los responsables académicos de los diferentes programas de pregrado en modalidad virtual y con el apoyo de la Asociación de Educación Superior con Programas a Distancia (ACESAD), contactados previamente por los responsables de este estudio para obtener una colaboración activa de estos, en aras de garantizar la aleatoriedad y la misma probabilidad de selección de cada uno de los miembros de la población estudiada. Del total de estudiantes que respondieron la encuesta se seleccionó una muestra de 384 estudiantes $(1-\alpha=95 \%$ y e=+/-5) de los 1098 que han hecho parte de este estudio.

\section{Instrumentos}

Para la obtención de información se ha implementado un cuestionario elaborado ad hoc a partir de los modelos de abandono enunciados por Cabrera et al. (2006), DeLone y McLean (1992, 2003), Salam y Farooq (2020) 
y Tinto (1987), entre otros. El instrumento diseñado para el abordaje del tema propuesto, consta de una primera pregunta sobre las razones de abandono (con seis opciones de respuesta) que va a permitir obtener información sobre las categorías de la variable dependiente en el análisis multinomial y 39 preguntas, asociadas a: variables de tipo sociodemográfico (edad, departamento, zona, sexo, estado civil, etnia o grupo), socioeconómico (situación laboral, salario, número de hijos, vivienda, estrato, ayudas sociales, entre otras), académica (año de graduación y titularidad del centro, motivos de elección de la carrera, modalidad preferida, entre otras), y otras asociadas a la infraestructura tecnológica para el desarrollo de este tipo de programas (lugar y calidad de conexión, dispositivo de entrada en la plataforma, entre otros), así como variables relacionadas con la valoración que los estudiantes hacen del contenido, perfil del profesorado, relaciones con compañeros, institución y plataforma a través de una escala de 1 (totalmente en desacuerdo) a 5 (totalmente de acuerdo) puntos.

La encuesta ha sido validada por especialistas en el área de la educación superior y sometida a una prueba piloto lanzada en julio de 2019, recogiendo información de un 10\% de la muestra total, permitiendo verificar la consistencia interna del instrumento con un $\alpha$ de Cronbach de .944.

La aplicación del instrumento final de la encuesta se llevó a cabo durante el último semestre de 2019 y el primer trimestre de 2020, y obtuvo una confiabilidad alta, tanto en la escala total $(\alpha=.941)$, como en el análisis de las subescalas $(\alpha=$ entre .842 y .917).

Los valores de asimetría, curtosis y la prueba Kolmogorov-Smirnov (K-S) con un valor p inferior a .05 para cada uno de los elementos de la escala indican la alta dispersión de las valoraciones hechas por los estudiantes y por ello siguiendo a autores como Fabrigar et al. (1999), se ha optado por emplear el método de mínimos cuadrados generalizados (GLS) como alternativa al de máxima verosimilitud (ML), de esta manera es posible seguir con el Análisis Factorial Exploratorio (AFE) para las 23 preguntas de la escala (mostrados en la tabla 2), empleando la rotación oblicua Promax que, según recientes estudios, ofrece resultados más robustos que otros métodos de rotación ortogonal (Panaretos et al., 2021). El AFE mostró que las 23 preguntas de la escala saturaron en cuatro componentes explicando el $68.9 \%$ de varianza del fenómeno estudiado (tabla 1 ).

Los valores observados en el índice Kaiser-Meyer-Olkin (KMO =.921) y en la prueba de esfericidad de Bartlett $\left(\mathrm{X}^{2}=6395.691\right.$, $\left.\mathrm{gl}=253, \mathrm{p}<.001\right)$, nos muestran niveles adecuados para poder realizar el AFE (Correa et al., 2006). Ello, al evidenciar la existencia de cuatro factores latentes independientes (tabla 1): "Calidad del sistema" (F1), "Calidad del servicio" (F2), "Calidad de 
la información" (F3) y "Calidad de las interacciones" (F4), relacionadas a las variables representadas en la tabla 2.

\section{Tabla 1}

Varianza explicada (autovalores iniciales)

\section{Autovalores iniciales}

\begin{tabular}{ccccccc}
\hline Factor & Total & $\begin{array}{c}\% \text { de } \\
\text { varianza }\end{array}$ & $\begin{array}{c}\% \\
\text { acumulado }\end{array}$ & Total & $\begin{array}{c}\% \text { de } \\
\text { varianza }\end{array}$ & $\begin{array}{c}\% \\
\text { acumulado }\end{array}$ \\
\hline 1 & 10.174 & 44.236 & 44.236 & 9.455 & 41.107 & 41.107 \\
\hline 2 & 2.444 & 10.625 & 54.860 & 1.969 & 8.561 & 49.667 \\
\hline 3 & 1.759 & 7.647 & 62.507 & 2.093 & 9.100 & 58.767 \\
\hline 4 & 1.464 & 6.366 & 68.873 & 1.264 & 5.494 & 64.261 \\
\hline
\end{tabular}

Método de extracción: cuadrados mínimos generalizados

\section{Tabla 2}

Matriz de componente rotado Promax con normalización Kaiser

\begin{tabular}{lcccc}
\hline & \multicolumn{5}{c}{ Factor } \\
\hline & $\mathbf{1}$ & $\mathbf{2}$ & $\mathbf{3}$ & $\mathbf{4}$ \\
\hline Materiales dispuestos en la plataforma & $\mathbf{. 8 9 2}$ & .405 & .463 & .3 \\
\hline Navegación de la plataforma & $\mathbf{. 8 9 1}$ & .447 & .431 & .244 \\
\hline Usabilidad de la plataforma & $\mathbf{. 8 5 5}$ & .478 & .46 & .269 \\
\hline Disposición de vídeos en plataforma & $\mathbf{. 8 2 1}$ & .419 & .473 & .308 \\
\hline Conectividad de la plataforma & $\mathbf{. 7 9 6}$ & .411 & .408 & .255 \\
\hline Diseño de la plataforma & $\mathbf{. 7 0 6}$ & .54 & .488 & .316 \\
\hline Herramientas de comunicación dis- & $\mathbf{. 6 9 1}$ & .601 & .484 & .375 \\
puestas en plataforma & & & & \\
\hline Vías de comunicación institucional & .45 & $\mathbf{. 8 4 2}$ & .502 & .473 \\
\hline Procesos administrativos & .448 & $\mathbf{. 7 7 6}$ & .505 & .337 \\
\hline Comunicación con el mentor & .325 & $\mathbf{. 7 7}$ & .418 & .326 \\
\hline Resolución de dudas del docente & .38 & $\mathbf{. 7 3 4}$ & .669 & .435 \\
\hline Plataforma asesoramiento & .464 & $\mathbf{. 7 3 3}$ & .494 & .375 \\
\hline Contacto con docente & .356 & $\mathbf{. 7 1 9}$ & .664 & .488 \\
\hline Bienestar & .459 & $\mathbf{. 7 1 8}$ & .509 & .423 \\
\hline Contenido útil & .468 & .47 & $\mathbf{. 8 7 2}$ & .291 \\
\hline Contenido suficiente & .419 & .518 & $\mathbf{. 8 3 2}$ & .328 \\
\hline
\end{tabular}




\begin{tabular}{ccccc}
\hline & \multicolumn{5}{c}{ Factor } \\
\hline Contenido claro & $\mathbf{1}$ & $\mathbf{2}$ & $\mathbf{3}$ & $\mathbf{4}$ \\
\hline Contenido actual & .48 & .552 & $\mathbf{. 8 2 6}$ & .336 \\
\hline Conocimiento del docente & .408 & .507 & $\mathbf{. 7 8 9}$ & .372 \\
\hline Acceso a biblioteca & .411 & .58 & $\mathbf{. 6 4 3}$ & .355 \\
\hline Contacto con compañeros & .524 & .6 & $\mathbf{. 6 3 1}$ & .384 \\
\hline Trabajo cooperativo & .31 & .409 & .372 & $\mathbf{. 9 7 1}$ \\
\hline Amistad con los compañeros & .301 & .453 & .402 & $\mathbf{. 8 1 6}$ \\
\hline
\end{tabular}

Método de extracción: cuadrados mínimos generalizados

Método de rotación: Promax con normalización Kaiser

\section{Análisis de datos}

Para cumplir con el objetivo señalado al inicio del estudio, se trabajó con el software SPSS, versión 22 para Windows, a partir de un modelo de regresión logística multinomial. Con este modelo, es posible determinar los efectos de las variables explicativas recogidas en la encuesta sobre la variable dependiente (razones de abandono), a través de las seis opciones de respuesta consideradas en esta (1: Obligaciones Laborales, 2: Problemas para pagar los estudios, 3: Bajo rendimiento/Dificultades con la Institución, 4: Compromisos personales/familiares, 5: Falta de accesibilidad, 6: Carrera/ Programa no interesante).

En el modelo se han empleado por un lado las variables demográficas, socioeconómicas, académicas y de infraestructura recogidas en el instrumento que han demostrado su relación con $\mathrm{Y}=\mathrm{j}$, y por otro lado las variables relativas a la satisfacción agrupadas en los factores latentes independientes entre ellas (tabla 2).

Para la identificación del modelo de regresión logística más adecuado, en función de las características del estudio, se han comparado diferentes modelos basados en el coeficiente de verosimilitud determinando que, por la cantidad de variables recogidas, el más adecuado es el personalizado a través del método de pasos sucesivos hacia adelante adoptando el principio de parsimonia y seleccionando únicamente las variables que mejor explican la variable dependiente (Pérez, 2014). Se ha tomado como categoría de referencia para el análisis la tercera (Bajo rendimiento/Dificultades con la Institución) al ofrecer una mayor explicación de las variables independientes. 


\section{RESULTADOS}

El análisis descriptivo de los datos determina la significativa presencia femenina con un $57 \%$ frente al $43 \%$ de hombres. Estudiantes en su mayoría adultos (el $72 \%$ es mayor de 31 años) que compaginan el desarrollo de su carrera académica con el desempeño profesional (88\%) y el cuidado familiar (el $65.70 \%$ tiene hijos a cargo), respondiendo a un perfil no tradicional identificado por autores como Sánchez-Gelabert y Elias (2017).

A nivel geográfico se reproduce la problemática señalada en el marco teórico, anteriormente abordado, pudiendo advertir la diferencia entre los porcentajes de estudiantes que provienen de núcleos urbanos $(91 \%)$ frente a los que provienen de rurales $(9 \%)$ y destacando la concentración de estudiantes en los departamentos con mayor desarrollo como Bogotá D.C (36.72\% de estudiantes), junto con Cundinamarca (11.46\%), Antioquia (8.59\%) y Valle del Cauca (7.29\%), encontrando una baja representación de estudiantes que provienen de las zonas con menos oferta académica y mayor dificultad de acceso sobre todo de las regiones Amazónica y Orinoquía.

En la muestra se encuentra cómo la mayoría de los estudiantes cuentan con salarios bajos (solamente el 29\% supera los $\$ 2000000$ mensuales), son beneficiarios de ayudas sociales (49\%) y solamente el 34\% posee vivienda propia. En su mayoría estos estudiantes han realizado sus estudios de educación media en centros de titularidad pública (75\%) mientras que para acceder a la educación superior han optado por una institución privada (93\% de los encuestados).

En el contexto de los datos analizados cabe mencionar que las principales razones de abandono tienen relación con el ámbito económico en cuanto a la imposibilidad de acometer el pago de los estudios iniciados, seguido por los problemas relacionados con el bajo rendimiento o dificultades con la institución (figura 1).

Para definir el modelo sobre el que va a girar este estudio se obtienen los estadísticos de la información de ajuste del modelo con -2 log de la verosimilitud (tabla 3) y Chi-cuadrado de la bondad de ajuste de Pearson y de la desviación que presentan p-valores aceptables (tabla 4), así como los valores aceptables de la R2 de Cox y Snell, de Nagerlkerke y de McFadden (tabla 5) y las pruebas de la razón de verosimilitud para los efectos del modelo y los parciales (tabla 6) cuyos p-valores por debajo de 0.05 muestran una alta significatividad. Todos estos datos permiten explicar que el $49.3 \%$ de las observaciones resultan correctamente clasificadas por el modelo con un alto porcentaje de explicación en las razones "Problemas para pagar los 
estudios" (67.70\%) y "Bajo rendimiento o Dificultades con la Institución: comunicación, atención, seguimiento" (56.80\%).

\section{Figura 1}

Razones de abandono

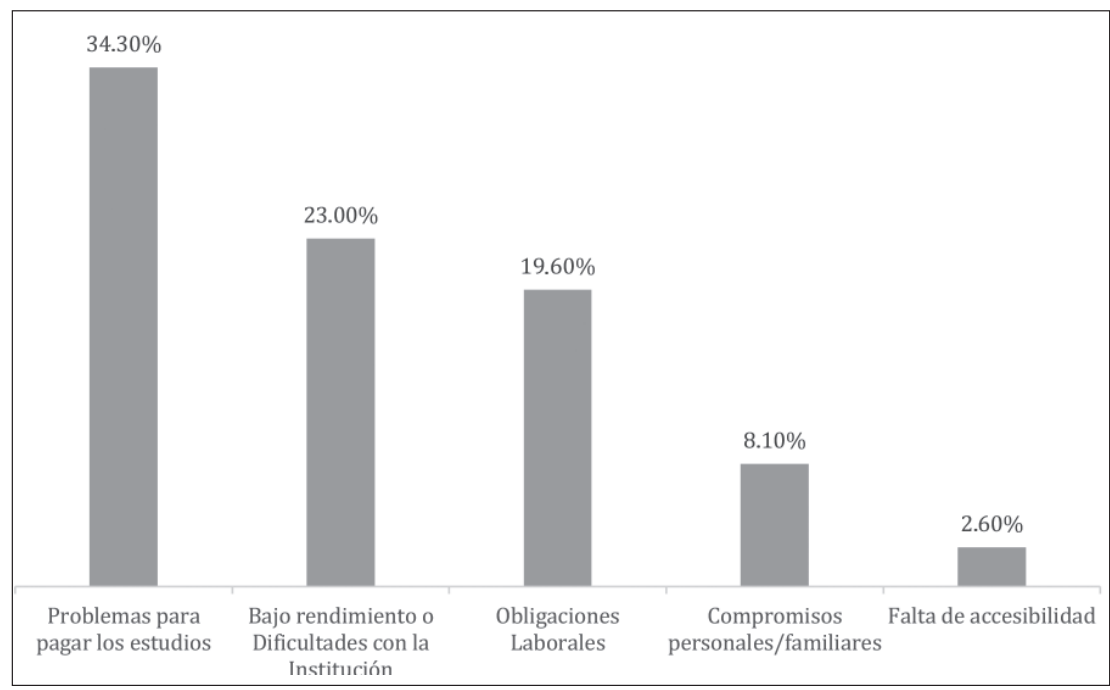

Elaboración propia

\section{Tabla 3}

Información de ajuste de los modelos

\section{Criterios de ajuste de modelo \\ Contraste de la razón de verosimilitud}

\begin{tabular}{lccccccc}
\hline \multicolumn{1}{c}{ Modelo } & AIC & BIC & $\begin{array}{c}\text { Logaritmo } \\
\text { de la } \\
\text { verosimilitud } \\
-\mathbf{2}\end{array}$ & $\begin{array}{c}\text { Chi- } \\
\text { cuadrado }\end{array}$ & gl & Sig. \\
\hline Solo interceptación & 1186.229 & 1205.810 & 1176.229 & & & \\
\hline Final & 1116.232 & 1409.947 & 966.232 & 209.997 & 70 & .000 \\
\hline
\end{tabular}

\section{Tabla 4}

Bondad de ajuste

\begin{tabular}{lccc}
\hline & Chi-cuadrado & gl & Sig. \\
\hline Pearson & 1689.760 & 1770 & .913 \\
\hline Desvianza & 964.846 & 1770 & 1.000 \\
\hline
\end{tabular}




\section{Tabla 5}

Pseudo R cuadrado

\begin{tabular}{ll}
\hline Cox y Snell & .432 \\
\hline Nagelkerke & .451 \\
\hline McFadden & .178 \\
\hline
\end{tabular}

\section{Tabla 6}

Contraste de la razón de verosimilitud

\begin{tabular}{lrrrrrrr}
\hline & \multicolumn{6}{c}{ Criterios de ajuste de modelo } & $\begin{array}{c}\text { Contraste de la razón de } \\
\text { verosimilitud }\end{array}$ \\
\cline { 2 - 9 } Efecto & $\begin{array}{c}\text { AIC de } \\
\text { modelo } \\
\text { reduci- } \\
\text { do }\end{array}$ & $\begin{array}{c}\text { BIC de } \\
\text { modelo } \\
\text { reduci- } \\
\text { do }\end{array}$ & $\begin{array}{c}\text { Logarit- } \\
\text { mo de la } \\
\text { verosimi- } \\
\text { litud -2 de } \\
\text { modelo } \\
\text { reducido }\end{array}$ & $\begin{array}{c}\text { Chi-cuadra- } \\
\text { do }\end{array}$ & gl & Sig. \\
\hline Interceptación & 1116.232 & 1409.947 & 966.232 & & 0 & 0 & \\
\hline ValServicio & 1121.703 & 1395.837 & 981.703 & 15.471 & 5 & .009 \\
\hline Calidad_conexion & 1120.104 & 1394.238 & 980.104 & 13.871 & 5 & .016 \\
\hline Salario & 1117.941 & 1313.751 & 1017.941 & 51.709 & 25 & .001 \\
\hline Sisben & 1124.76 & 1379.313 & 994.76 & 28.528 & 10 & .001 \\
\hline Hijos & 1119.538 & 1393.672 & 979.538 & 13.305 & 5 & .021 \\
\hline Horas_estudio_dia & 1118.451 & 1373.004 & 988.451 & 22.218 & 10 & .014 \\
\hline ValContenido & 1122.107 & 1396.241 & 982.107 & 15.874 & 5 & .007 \\
\hline Vulnerable & 1118.236 & 1392.37 & 978.236 & 12.003 & 5 & .035 \\
\hline
\end{tabular}

El estadístico de chi-cuadrado es la diferencia de los logaritmos de la verosimilitud -2 entre el modelo final y el modelo reducido. El modelo reducido se forma omitiendo un efecto del modelo final. La hipótesis nula es que todos los parámetros de dicho efecto son 0 .

a. Este modelo reducido es equivalente al modelo final porque omitir el efecto no aumenta los grados de libertad.

b. Se han encontrado singularidades inesperadas en la matriz hessiana. Esto indica que o bien se deben excluir algunas variables de predictor, o bien se deben fusionar algunas categorías.

Después de evaluar la significancia global del modelo y confirmar la bondad de ajuste a través de los diferentes criterios señalados anteriormente, en la tabla 7 se estiman las k-1 ecuaciones, en las que $\mathrm{k}$ es el número de niveles que adopta la variable dependiente. Al analizar la ecuación 1 (Obligaciones laborales), se aprecia que son estadísticamente significativos la valoración del servicio ofrecido por la institución y las horas de estudio, de tal manera, que la probabilidad de abandonar los estudios debido a las 
obligaciones laborales es mayor cuando la valoración del servicio prestado por la institución no es el óptimo y además cuando la dedicación diaria al estudio es baja.

En la ecuación 2 (Problemas para pagar los estudios) es un 3.38\% (1/29.587) más probable abandonar los estudios cuando el salario percibido por los estudiantes se encuentra entre los $\$ 250621$ y $\$ 828116$ y un $17.42 \%$ cuando el rango salarial está entre $\$ 828117$ y $\$ 1000000$. A medida que el salario percibido aumenta, la probabilidad de abandonar por razones económicas disminuye. En esta ecuación destaca cómo la probabilidad de abandonar los estudios es mayor al 51\% cuando la valoración del servicio y de la información obtenida para el desarrollo del programa no se corresponde con el nivel deseado.

De nuevo se repite la alta probabilidad (33\%) de abandonar los estudios por "Compromisos personales/familiares" si los estudiantes no tienen una valoración positiva del servicio prestado por la institución para el desarrollo del programa formativo. Mientras que cuando es por "Falta de accesibilidad" es la condición de vulnerabilidad de los estudiantes la que aumenta el riesgo. Pertenecer a alguno de los grupos con mayores desventajas acumulativas como la población desplazada y víctima del conflicto armado, población con discapacidad, grupos étnicos, indígenas, comunidades negras, Rom, población rural dispersa y población frontera aumenta en 12.3 veces la probabilidad de abandonar los estudios. Además de lo anterior, poseer una calidad de conexión baja aumenta el riesgo de abandonar los estudios por falta de accesibilidad y también lo incrementa el tener hijos.

En la ecuación 5 (Carrera/programa no interesante), el pertenecer a alguno de los grupos vulnerables anteriormente mencionados o estar registrado en el Sistema de Selección de Beneficiarios Para Programas Sociales de Colombia (SISBEN), aumenta la probabilidad de abandonar el programa en 2.8 y 14.3 veces respectivamente. También la baja valoración del servicio o la baja dedicación diaria al estudio aumentan la probabilidad de abandonar el programa formativo.

A la luz de los resultados alcanzados en cada una de las ecuaciones es posible hacer una valoración de las hipótesis planteadas de tal manera que se admite la $\mathrm{H} 1$ y $\mathrm{H} 2$ que hacen referencia a la asociación establecida entre el abandono de los estudios y las variables socioeconómicas y sociodemográficas de los estudiantes al encontrar como el bajo salario o la necesidad de beneficiarse de programas sociales como el SISBEN están determinando la posibilidad de abandonar los estudios o variables como la pertenencia a un grupo vulnerable, la tenencia de hijos o la dedicación a los estudios también determinan la posibilidad de abandonar el programa. 
La valoración que los estudiantes realizan del servicio prestado por la institución se ha definido como un elemento clave en la probabilidad de abandonar los estudios permitiendo admitir la tercera hipótesis planteada (H3) y también la quinta (H5) que relaciona la probabilidad de abandonar el programa con la calidad de la información ofrecida por la institución. No sucede lo mismo con las hipótesis $\mathrm{H} 4$ y H6 para las que no se han encontrado evidencias que relacionen el abandono y calidad de la infraestructura tecnológica prestada o la calidad de las interacciones entre pares y docentes.

\section{CONCLUSIONES Y DISCUSIÓN}

El abandono educativo en Colombia es una realidad compleja en la que intervienen factores diversos. A pesar de las limitaciones propias del estudio presentado, donde la muestra está acotada a las 384 unidades analizadas, no existen argumentos suficientes para rechazar las hipótesis H1, H2, H3 y H5, a nivel de la población estudiada, no sucediendo lo mismo con las hipótesis $\mathrm{H} 4$ y H6, planteadas en este trabajo. La consideración de estas hipótesis posibilita conocer los factores que repercuten de manera directa en la decisión de abandonar los programas y puede contribuir de manera positiva en la orientación de políticas educativas e institucionales que atiendan las necesidades encontradas en el estudio, y que permitan alcanzar los objetivos de cobertura, permanencia y graduación.

Uno de los elementos detectados en el contexto educativo virtual en Colombia, es que la calidad del servicio ofrecido por la institución para el desarrollo de los programas formativos resulta clave en la decisión de permanencia de los estudiantes. Una calidad donde se prioriza la necesidad de establecer canales comunicativos a través del servicio de mentoría y la relación con los docentes y tutores para la resolución de dudas e inquietudes surgidas durante el proceso académico, además de la disposición de los medios adecuados para satisfacer las necesidades académicas de los estudiantes (programas de bienestar estudiantil, procesos administrativos, apoyo técnico, etc.). Algo que cobra especial relevancia en entornos online donde la mediación entre los estudiantes y la institución educativa se realiza a través de las herramientas y canales comunicativos virtuales dispuestos por la universidad, y donde la percepción de la calidad del servicio puede influir de manera decisiva en los niveles de satisfacción de los estudiantes y afectar a sus intenciones de comportamiento (permanencia o deserción) (Segovia-García y Said-Hung, 2021).

La calidad de servicio también se ha contemplado como clave en otros estudios como los desarrollados por Al-Fraihat et al. (2020) y Mustafá et al. (2020), donde se identifica la necesidad de establecer una relación entre el 
estudiante y la institución que sea óptima y que reduzca la sensación de soledad, falta de empatía o falta de respuesta ante los problemas planteados ejerciendo así una influencia positiva en la satisfacción.

Junto con lo anterior es necesario también destacar la importancia otorgada a la calidad del contenido con el que trabajan los estudiantes en su programa. La repercusión de contar con un contenido actualizado, de calidad, útil y suficiente junto con la provisión de herramientas adecuadas para complementarlo (bases bibliográficas, material complementario, casos de éxito, entre otros), así como el pertinente conocimiento de los docentes que acompañan en el proceso formativo son elementos que también pueden determinar la intención de abandono. Y este factor, la calidad del contenido, adquiere un peso fundamental cuando el abandono se debe a "Problemas para pagar los estudios" reforzando aún más la obligación de que las universidades se esfuercen en ofrecer información de calidad y bien organizada para cumplir con las expectativas de los estudiantes para los que permanecer en el programa puede suponer un esfuerzo importante. Este resultado coincide con los aportados por Al-Fraihat et al. (2020) y Mustafá et al. (2020), quienes advierten cómo un contenido irrelevante, inexacto, incorrecto o inoportuno disminuye la percepción de calidad sobre la información, pero también sobre el servicio ofertado redundando de manera directa en la percepción de los estudiantes y su satisfacción y de manera indirecta en el impacto que puede tener en el comportamiento de los estudiantes (permanencia, dedicación, disposición de pago, etc.). Es por lo que las IES, deberían priorizar estrategias que permitan valorar críticamente la calidad del servicio e información que están ofreciendo a sus estudiantes, garantizando que los contenidos con los que se trabaja estén actualizados y sean pertinentes con los resultados de aprendizaje que se esperan conseguir.

Dentro de los factores socioeconómicos destaca, con una presencia muy significativa, el salario como variable que determina la decisión de abandono y que como se ha visto anteriormente en interacción con otros factores asociados a la satisfacción con la institución y su servicio pueden ser determinantes a la hora de tomar esta decisión encontrando que los estudiantes con menor poder adquisitivo y que en algunos casos son beneficiarios de ayudas sociales como las del SISBEN, presentan una mayor probabilidad de abandonar los estudios. Este resultado permite reafirmar las tesis de Melguizo et al. (2016) que indican cómo las desventajas en cuanto a poder adquisitivo o dificultades económicas son elementos que repercuten directamente en el abandono de los estudios. Un factor, el económico, que cobra una importancia crucial en estos momentos de crisis donde además de los problemas sanitarios generados por la pandemia se están desencadenando otros de índole económico y social derivados de la 
incertidumbre en los mercados bursátiles mundiales. Algo que parece crucial y con consecuencias aún no determinadas en países de América Latina y el Caribe, como Colombia, donde tal como recoge Weller (2020) el impacto en el mercado laboral será importante y la pérdida de empleos, la falta de seguros sociales y protección pueden agravar la situación de pobreza para muchas personas y más cuando los datos de empleo apuntan en este país una caída que podría llegar hasta el 20.5\% aumentando la vulnerabilidad entre poblaciones donde se hace difícil asegurar los ingresos (Mejía, 2020; Programa de las Naciones Unidas para el Desarrollo [PNUD], 2020). Lo anterior puede impactar en la posibilidad de permanencia de muchos estudiantes y más aún cuando las estrategias desplegadas por el MEN para paliar los efectos de la crisis se orientan hacia el fomento de ayudas al pago de matrículas para estudiantes de pregrado en situación de vulnerabilidad pero únicamente de las 63 IES públicas (MINEDUCACIÓN, 2021) reforzando la brecha ya señalada por Bonilla et al., (2015) y Gamboa y RodríguezLesmes (2014), para aquellos alumnos en situación de vulnerabilidad que por falta de cupo o condiciones de acceso han tenido que acudir a un centro de titularidad privada.

Esta situación de vulnerabilidad ya es una variable significativa en el momento de realizarse el estudio, encontrando que la pertenencia a un grupo con mayores desventajas acumulativas se registra como un predictor del abandono de la educación superior, sobre todo cuando existen problemas por "Falta de accesibilidad" o por inscribirse en una "Carrera/programa no interesante". Pertenecer a grupos étnicos, víctimas del conflicto armado, discapacitados, población ROM, habitantes fronteras, campesinos, población LGTBI, entre otros, puede aumentar el riesgo de abandono educativo si no se ofrece una respuesta educativa plural y acorde con las necesidades de los estudiantes. De nuevo se circunscribe esta variable a la necesidad de generar procesos de evaluación en las IES donde se analice de manera constructiva la idoneidad y calidad del servicio e información prestado en aras de garantizar espacios académicos adecuados para que todos los estudiantes participen de su proceso formativo con plena autonomía y sin limitaciones. En este sentido las IES pueden encontrar en la tecnología una herramienta de un gran valor para desarrollar estrategias pedagógicas y diseñar metodologías que apuesten por respetar esta pluralidad que se presenta en las aulas, respetando los diferentes estilos y ritmos de aprendizaje, facilitando y enriqueciendo la información a través de múltiples canales y formatos, generando espacios de trabajo donde los estudiantes puedan seleccionar la manera que más les interese para acercarse al conocimiento en función de sus necesidades, motivaciones o tiempo de dedicación.

Se apuesta así por un Diseño Universal de Aprendizaje (DUA) (AlAzawei et al., 2017), donde se faciliten múltiples formas de representación 
de la información, diferentes alternativas de expresión y se respeten diversas vías de implicación en el proceso formativo, permitiendo adaptar su trabajo personal en función de la disponibilidad horaria y permitiendo compatibilizar esta responsabilidad con otras obligaciones como el cuidado de los hijos o el trabajo.

Finalmente, y aunque la calidad de la infraestructura tecnológica no se ha registrado como un factor significativo, es necesario advertir que Colombia es uno de los países de América Latina y el Caribe con peor conectividad (Comisión Económica para América Latina y el Caribe, 2016; CAF, 2020), y esta deficiencia repercute directamente en los usuarios. En este sentido, las IES deberían tener en cuenta las dificultades de acceso que pueden experimentar algunos alumnos debido a la mala calidad de sus conexiones y facilitar unas plataformas de formación y recursos que sean accesibles. Garantizar una adecuada navegación por el entorno de formación con materiales fácilmente descargables, la posibilidad de visualizar los contenidos offline desde cualquier dispositivo mejorará la experiencia de usuario y redundará en la permanencia de los estudiantes.

Los factores detectados en este estudio pueden contribuir a enriquecer el conocimiento sobre el abandono de la educación superior en modalidad virtual. Algunos factores, como los socioeconómicos y sociodemográficos, han sido ampliamente estudiados, pero otros como la calidad del servicio y la calidad de la información requieren de una revisión más profunda. Se abren nuevas líneas de investigación donde debería implicarse no solamente a los estudiantes para conocer cuáles son sus expectativas y previsiones cuando se inscriben en programas de modalidad virtual y la satisfacción experimentada cuando los reciben, sino también a las universidades, sus equipos docentes y directivos para analizar su percepción sobre el servicio ofrecido y contrastar puntos de vista que ayuden a orientar respuestas educativas plurales que permitan atender de manera satisfactoria a todos los estudiantes. 


\section{REFERENCIAS BIBLIOGRÁFICAS}

Al-Azawei, A., Parslow, P., \& Lundqvist, K. (2017). The effect of Universal Design for Learning (UDL) Application on e-learning acceptance: A structural equation model. International Review of Research in Open and Distributed Learning, 18(8), 54-87. https://doi. org/10.19173/irrodl.v18i6.2880

Aldholay, A., Isaac, O., Abdullah, Z., Abdulsalam, R., \& Al-Shibami, A. H. (2018). An extension of Delone and McLean IS success model with selfefficacy. The International Journal of Information and Learning Technology, 35(4), 285-304. https://doi.org/10.1108/ ijilt-11-2017-0116

Al-Fraihat, D., Joy, M., Masa'deh, R., \& Sinclair, J. (2020). Evaluating E-learning systems success: An empirical study. Computers in Human Behavior, 102, 67-86. https://doi. org/10.1016/j.chb.2019.08.004

Arias-Velandia, N., Rincón-Báez, W. U., \& Cruz-Pulido, J. M. (2018). Desempeño de mujeres y hombres en educación superior presencial, virtual y a distancia en Colombia. Panorama, 12(22), 57-69. https://doi.org/10.15765/ pnrm.v12i22.1142

Benavides, M., Arellano, A., \& Zárate, J. S. (2018). Market- and governmentbased higher education reforms in Latin America: the cases of Peru and Ecuador, 2008-2016. Higher Education, 77(6), 1015-1030. https:// doi.org/10.1007/s10734-018-0317-3

Bonilla, L., Bottan, N. L., \& Ham, A. (2015). The role of information on students' career choice and school effort experimental evidence from Bogotá, Colombia. SSRN Electronic Journal, 1-40. https://doi.org/10.2139/ ssrn. 2546835
Cabrera, L., Bethencourt, J. T., Pérez, P. \& González, M. (2006). El problema del abandono de los estudios universitarios. Relieve, 12(2), 171-203. https://doi.org/10.7203/ relieve.12.2.4226 https://n9.cl/8yyt

CAF (2020). Las oportunidades de la digitalización en América Latina frente al Covid-19.

Comisión Económica para América Latina y el Caribe (CEPAL) (2016). Estado de la banda ancha en América Latina y el Caribe 2016. http://bit. ly/38Geihx

Correa, J., Iral, R., \& Rojas, L. (2006). Estudio de potencia de pruebas de homogeneidad de varianza. Revista colombiana de estadística, 29(1), 5776. https://cutt.ly/RbPuS3P

DeLone, W. H., \& McLean, E. R. (1992). Information systems success: The quest for the dependent variable. Information Systems Research, 3(1), 60-95. https://doi.org/10.1287/ isre.3.1.60

DeLone, W. H., \& McLean, E. R. (2003). The DeLone and McLean model of information systems success: A tenyear update. Journal of Management Information Systems, 19(4), 9-30. https://doi.org/10.1080/07421222.2003 .11045748

Fabrigar, L. R., Wegener, D. T., MacCallum, R. C., \& Strahan, E. J. (1999). Evaluating the use of exploratory factor analysis in psychological research. Psychological Methods, 4(3), 272-299. https://doiorg.ezproxy.uned.es/10.1037/1082989X.4.3.272.

Fernández, J. (2016). Alumnado inmigrante en la eso: vulnerabilidad 
pedagógica del sistema educativo. Educación XX1, 20(1), 121-140. https:// doi.org/10.5944/educxx1.17494

Fernández, N. R., \& Pérez, C. G. (2016). La educación superior latinoamericana en el inicio del nuevo siglo. Situación, principales problemas y perspectivas futuras. Revista Española de Educación Comparada, (27), 123-148. https://doi. org/10.5944/reec.27.2016.15044

Gamboa, L. F., \& Rodriguez-Lesmes, P. A. (2014). Do Colombian students underestimate higher education returns? Universidad del Rosario, Facultad de Economía. https://n9.cl/hv91s

Mejía, L. F. (2020). COVID-19: costos económicos en salud y en medidas de contención para Colombia. Fedesarrollo. https://bit.ly/31NTzGJ

Melguizo, T., Sanchez, F., \& Velasco, T. (2016). Credit for Low-Income Students and Access to and Academic Performance in Higher Education in Colombia: A Regression Discontinuity Approach. World Development, 80, 61-77. https://doi.org/10.1016/j. worlddev.2015.11.018

Ministerio de Educación Nacional (2010). Plan sectorial 2010-2014. https:/cutt.ly/wy1VdAZ

Ministerio de Educación Nacional (2021). Plan Estratégico Institucional 2019-2022. https://cutt.ly/TzPULo0

Munizaga, F. R., Cifuentes, M. B., \& Beltrán, A. J. (2018). Retención y abandono estudiantil en la educación superior universitaria en América Latina y el Caribe: una revisión sistemática. education policy analysis archives, 26(61), 1-35. https://doi. org/10.14507/epaa.26.3348

Mustafá, S., Kar, A., \& Janssen, M. (2020). Understanding the impact of digital service failure on users:
Integrating Tan's failure and DeLone and McLean's success model. International Journal of Information Management, 53, 102119. https://doi. org/10.1016/j.ijinfomgt.2020.102119

Organización para la Cooperación y el Desarrollo Económico (2019). Trends Shaping Education 2019. https://doi. org/10.1787/trends_edu-2019-en

Panaretos, D., Tzavelas, G., Vamvakari, M., \& Panagiotakos, D. (2021). Investigating the repeatability of the extracted factors in relation to the type of rotation used, and the level of random error: A simulation study. Journal of Data Science, 18(2), 390-404. https://doi.org/10.6339/ jds.202004_18(2).0010

Pérez, C. (2014). Técnicas estadísticas predictivas con IBM SPSS. Garceta.

Pérez, R. (2017). Estado de naturaleza, anomia y derecho. Una reflexión desde América Latina. Dikaiosyne, 32, 71-95. https://cutt.ly/Uy9QoMq

Programa de las Naciones Unidas para el Desarrollo (2020). El coronavirus en Colombia: vulnerabilidad y opciones de política. https://cutt.ly/AbPbySd

Salam, M., \& Farooq, M.S. (2020). Does sociability quality of web-based collaborative learning information system influence students' satisfaction and system usage? International Journal of Educational Technology in Higher Education, 17(1), 1-39. https:// doi.org/10.1186/s41239-020-00189-z

Sánchez-Gelabert, A., \& Elias, M. (2017). Los estudiantes universitarios no tradicionales y el abandono de los estudios. Estudios sobre Educación, 32, 27-48. https://doi. org/10.15581/004.32.27-48

Segovia-García, N., \& Said-Hung, E.M. (2021). Factores de satisfacción de los 
alumnos en e-learning en Colombia. Revista Mexicana de Investigación Educativa, 26(89), 595-621.

Sistema Nacional de Información de la Educación Superior (2019). Número de inscripciones en programas de educación superior - Colombia 2018 [Conjunto de datos]. http://bit. ly/39EJcqu

Sistema Nacional de Información de la Educación Superior (s.f.). Consulta de Instituciones [Conjunto de datos]. https://n9.cl/tvdwi
Sistema para la Prevención y Análisis de la Deserción en las Instituciones de Educación Superior (s.f.). Deserción anual por programa y metodología [Conjunto de datos].

Tinto, V. (1987). Leaving College: Rethinking the Causes and Cures of Student Attrition. University Of Chicago Press.

Weller, J. (2017). Las transformaciones tecnológicas y su impacto en los mercados laborales. Naciones Unidas. https://n9.cl/ck2je 


\section{PERFIL ACADÉMICO Y PROFESIONAL DE LOS AUTORES}

Nuria Segovia-García. ORCID: https://orcid.org/0000-0003-4329-0987

Licenciada en pedagogía por la universidad de Salamanca y Doctora en Educación por la UNIR. Actualmente es directora de tecnología educativa de la Red Internacional de Universidades Virtuales, Red SUMMA y docente e investigador del grupo Sinergi@ Digital. Las principales áreas de investigación, desarrolladas en los últimos cinco años, se enmarcan en el estudio de las TIC aplicadas en Educación, metodologías e innovación educativa. E-mail: nuria.seggar@gmail. com

Elías Said-Hung. ORCID: https://orcid.org/0000-0002-0594-5906

Profesor titular de la Facultad de Educación, miembro del grupo de investigación PROCOMM y director del Máster Universitario de Educación Inclusiva e Intercultural de la Universidad Internacional de la Rioja (UNIR); además de ser presidente de la Asociación Ciencia, Tecnología y Sociedad (CITESOC). Las principales áreas de investigación, desarrolladas en los últimos cinco años, se enmarcan en el estudio de las TIC aplicadas en Educación, Redes Sociales y Medios Digitales. E-mail: elias.said@unir.net

Francisco José García Aguilera. ORCID: https://orcid.org/0000-0002-57647409

Doctor en Educación e investigador del Grupo de Innovación y Desarrollo Educativo Inclusivo (IDEI). Licenciado en Pedagogía por la Universidad de Málaga, Máster Universitario en Psicopedagogía por la UNIR. Acreditado como Profesor Contratado Doctor por ANECA, ha sido profesor de la Universidad de Málaga y la Universidad Internacional de La Rioja. Principales líneas estratégicas: Inclusión social, formación de formadores e innovación, TIC y e-learning. E-mail: francisco.garcia.a@unir.net

Fecha Recepción del Artículo: 27. Marzo. 2021

Fecha Modificación del Artículo: 15. Mayo. 2021

Fecha Aceptación del Artículo: 17. Mayo. 2021

Fecha Revisión para Publicación: 10. Septiembre. 2021 